INPLASY

PROTOCOL

To cite: Zhu et al. Efficacy and Safety of Traditional Chinese Moxibustion Therapy for Patients with Gastritis: a Protocol for Systematic Review and Meta-analysis. Inplasy protocol 2020100014. doi: 10.37766/inplasy2020.10.0014

Received: 05 October 2020

Published: 05 October 2020

Corresponding author: Qingyuan Zhu

15970404083@163.com

Author Affiliation:

Nanchang Hongdu Hospital of Traditional Chinese Medicine

Support: No funding.

Review Stage at time of this submission: The review has not yet started.

Conflicts of interest:

The authors declare no conflicts of interest.

\section{Efficacy and Safety of Traditional Chinese Moxibustion Therapy for Patients with Gastritis: a Protocol for Systematic Review and Meta-analysis}

Zhu, QY1; Hou, XJ2.

Review question / Objective: To evaluate the efficacy and safety of Traditional Chinese Moxibustion therapy for patients with Gastritis.

Condition being studied: Gastritis is one of the most common life-long, serious and insidious illnesses with such severe killing sequelae as peptic ulcer or gastric cancer in human beings. Globally, on average, even more than half of people may have a chronic gastritis at present and millions of premature deaths may occur annually worldwide due to cancer and ulcer as sequelae of the gastritis. Helicobacter pylori infection is the main cause of gastritis. A life-long and aggressive inflammation in gastritis results in destruction (atrophic gastritis) of stomach mucosa with time. Moxibustion, as one of the therapies in traditional Chinese medicine, has a certain relieving and therapeutic effect on the deterioration of gastritis, supported by a certain amount of research results.

INPLASY registration number: This protocol was registered with the International Platform of Registered Systematic Review and Meta-Analysis Protocols (INPLASY) on 05 October 2020 and was last updated on 05 October 2020 (registration number INPLASY2020100014).

\section{INTRODUCTION}

Review question / Objective: To evaluate the efficacy and safety of Traditional Chinese Moxibustion therapy for patients with Gastritis.
Condition being studied: Gastritis is one of the most common life-long, serious and insidious illnesses with such severe killing sequelae as peptic ulcer or gastric cancer in human beings. Globally, on average, 
even more than half of people may have a chronic gastritis at present and millions of premature deaths may occur annually worldwide due to cancer and ulcer as sequelae of the gastritis. Helicobacter pylori infection is the main cause of gastritis. A life-long and aggressive inflammation in gastritis results in destruction (atrophic gastritis) of stomach mucosa with time. Moxibustion, as one of the therapies in traditional Chinese medicine, has a certain relieving and therapeutic effect on the deterioration of gastritis, supported by a certain amount of research results.

\section{METHODS}

Participant or population: Patients with Gastritis (chronic gastritis, superficial gastritis and atrophic gastritis will be included).

Intervention: Moxibustion therapy such as direct moxibustion, indirect moxibustion, warm moxibustion, long snake moxibustion, Du-moxibustion, thunder-fire moxibustion and so on.

Comparator: Western medicine, placebo, sham acupuncture, no treatment, or any combination of these.

Study designs to be included: Randomized controlled trials (RCTs) which assessed the efficacy and safety of moxibustion therapy for gastritis will be included.

Eligibility criteria: Subjects: Patients with gastritis(type and stage of gastritis development, age and sex of patients were not restricted). Intervention measures: Moxibustion therapy like direct moxibustion, indirect moxibustion, warm moxibustion, long snake moxibustion, Dumoxibustion, thunder-fire moxibustion, etc. were used as the intervention measures in the treatment group, while conventional were used other therapy like western medicine, placebo, sham acupuncture, or any combination of these as the intervention measures in the control group Outcome measures: clinical effectiveness rate, clearance of $\mathrm{H}$-pylori infection, quality of life (SF-36), symptom scores (stomachache, stomach distention, belching, and acid reflux, etc), etc.

Information sources: China National Knowledge Infrastructure (CNKI), WangFang Database (WF), Chinese Scientific Journal Database(VIP), Chinese Biomedical Literatures Database(CBM). Pubmed, EMBASE, The Cochrane Library.

Main outcome(s): Clinical effectiveness rate which be divided into markedly effective, effective, or ineffective according to clinical symptoms.

Quality assessment / Risk of bias analysis: Quality assessment: According to the improved Jadad scoring scale, the quality of the included literature was evaluated. 1-3 were classified as low quality and 4-7 as high quality. Risk of bias assessment: The methodological quality of the RCTs will be independently assessed by two reviewers(ZQY/HXJ)according to the Cochrane Intervention System Review Manual (version 5.1.0). Seven areas will be considered and evaluated 3 levels ("Iow risk", "high risk" or "unclear risk") . Any disagreements will be resolved through discussion or consultation with a third reviewer(JYF)

Strategy of data synthesis: RevMan $\mathbf{5 . 4}$ software (Cochrane Collaboration) was used for the meta-analysis.Dichotomous data were reported as risk ratio (RR) with 95\% confidence intervals (CI), while continuous data were reported as standardized mean difference (SMD) with $95 \%$ Cls. The $I^{2}$ test was used to test heterogeneity with a significance level set at $\mathbf{5 0 \%}$. If heterogeneity was not significant $\left(I^{2} \leq 50 \%\right)$, the fixed-effect model was used for meta-analysis. Otherwise, the randomeffect model was used $\left(I^{2} \geq 50 \%\right)$. If possible, we investigated the potential explanations for heterogeneity and conducted subgroup analysis.

Subgroup analysis: If the necessary data are available, subgroup analysis will be 
carried out according to different factors as follows: 1. Control interventions (western medicine, placebo, sham acupuncture, no treatment, etc.). 2.Outcome indicators.

Sensibility analysis: Sensibility analysis: To assess the influence of each individual study, leave-one-out sensitivity analysis was performed iteratively by removing one study at a time to confirm that the findings were not influenced by any single study.

Country(ies) involved: China.

Keywords: chronic gastritis, superficial gastritis, atrophic gastritis, moxibustion, acupuncture, protocol, systematic review, Meta-analysis.

Contributions of each author:

Author 1 - Qingyuan Zhu - The author drafted and improved the manuscript.

Author 2 - Xinju Hou - The author provided statistical expertise. 